\title{
On the Design of Smart Home System
}

\author{
Muhanned AL-Rawi ${ }^{1}$, and Muaayed AL-Rawi ${ }^{2}$ \\ ${ }^{1}$ Ibb University, Yemen, E-mail: muhrawi@yahoo.com \\ ${ }^{2}$ University of AL-Mustansiryia, Iraq, E-mail: muaayed@yahoo.com
}

\begin{abstract}
Smart Home (SH) technology, comprising smart devices in the home context. SH technology has demonstrated new and good contribution to increase safety and reliability, at the same time; it might have its own influence to change our living habit. Use of mobile phones is changing with the development of technology; it can be used for different purposes. With the help of a network, mobile phone can be used to implement the smart home by controlling the devices. The objectives of this paper are to implement a smart home by controlling electronic devices at home remotely and to get an alert on intrusion or movement around the restricted premises. The mobile phone is using the short message service (SMS) service available and the alerts are also received as an SMS mentioning the activity occurring around the premises.
\end{abstract}

Keywords - smart home; mobile phone; design.

\section{INTRODUCTION}

$\mathrm{SH}$ is the term commonly used to define a residence that uses a home controller to integrate the various home automation systems. The most popular home controllers are those that are connected to a Windows based PC during programming only, and are then left to perform the home control duties on a standalone basis. Integrating the home systems allows them to communicate with one another through the home controller, thereby enabling single button and voice control of the various home systems simultaneously, in pre-programmed scenarios or operating modes. The home network encompasses communications, entertainment, security, convenience, and information systems.

Power line Carrier Systems (PCS) is a technology which is used to send coded signals along a home's existing electric wiring to programmable switches, or outlets. These signals convey commands that correspond to "addresses" or locations of specific devices, and that control how and when those devices operate. A PCS transmitter, for instance, can send a signal along a home's wiring, and a receiver plugged into any electric outlet in the home could receive that signal and operate the appliance to which it is attached. X10 is a common protocol for PCS. X10 signals, which involve short radio frequency (RF) bursts that represent digital information, enable communication between transmitters and receivers [1].

The European Installation Bus, or Instabus is an embedded control protocol for digital communication between smart devices. It consists of a two-wire bus line that is installed along with normal electrical wiring. The Instabus line links all appliances to a decentralized communication system and functions like a telephone line over which appliances can be controlled. The European Installation Bus Association is part of Konnex, an association that aims to standardize home and building networks in Europe [2].

All the appliances and devices are receivers. The means of controlling the system, such as remote controls or keypads, are transmitters. The transmitter message in numerical code includes the following [3]:

1. An alert to the system that it is issuing a command.

2. An identifying unit number for the device that should receive the command.

3. A code that contains the actual command, such as "turn off."

This all is to happen in less than a second, but X10 does have some limitations. Communicating over electrical lines is not always reliable because the lines get "noisy" from powering other devices. An X10 device could interpret electronic interference as a command and react, or it might not receive the command at all. But other technologies have emerged. Instead of using power lines, some systems use radio waves to communicate, which is also how $\mathrm{Wi}-\mathrm{Fi}$ and cell phone signals operate. However, home automation networks do not need all the juice of a Wi-Fi network because automation commands are short messages. The two most prominent radio networks in home automation are ZigBee and Z-Wave. Both of these technologies are mesh networks, meaning there is more than one way for the message to get to its destination [4].

\section{DESIGN OF SMART HOME SYSTEM}

The system mainly comprises two parts; the mobile station and the microcontroller unit. The mobile station is responsible for giving the command and the control instruction to the devices and sensors and to get the response from them. It is just a user interface and does not control the devices. The second unit, the microcontroller unit, is responsible for controlling the devices, processing information gathered from the devices as well as from the mobile station. The microcontroller unit is the brain of the system and controls and processes information to and from all other units of the system.

The block diagram of the system is shown in Fig. 1. The microcontroller unit consists of four sensors (the temperature detector, the digital output proximity sensor is the intrusion detector, the passive infrared sensor is the motion detector, and the heat sensor is responsible as far as a fire happened, it sends to us SMS), and a light control along with the sim900 module. The data from the sensors 


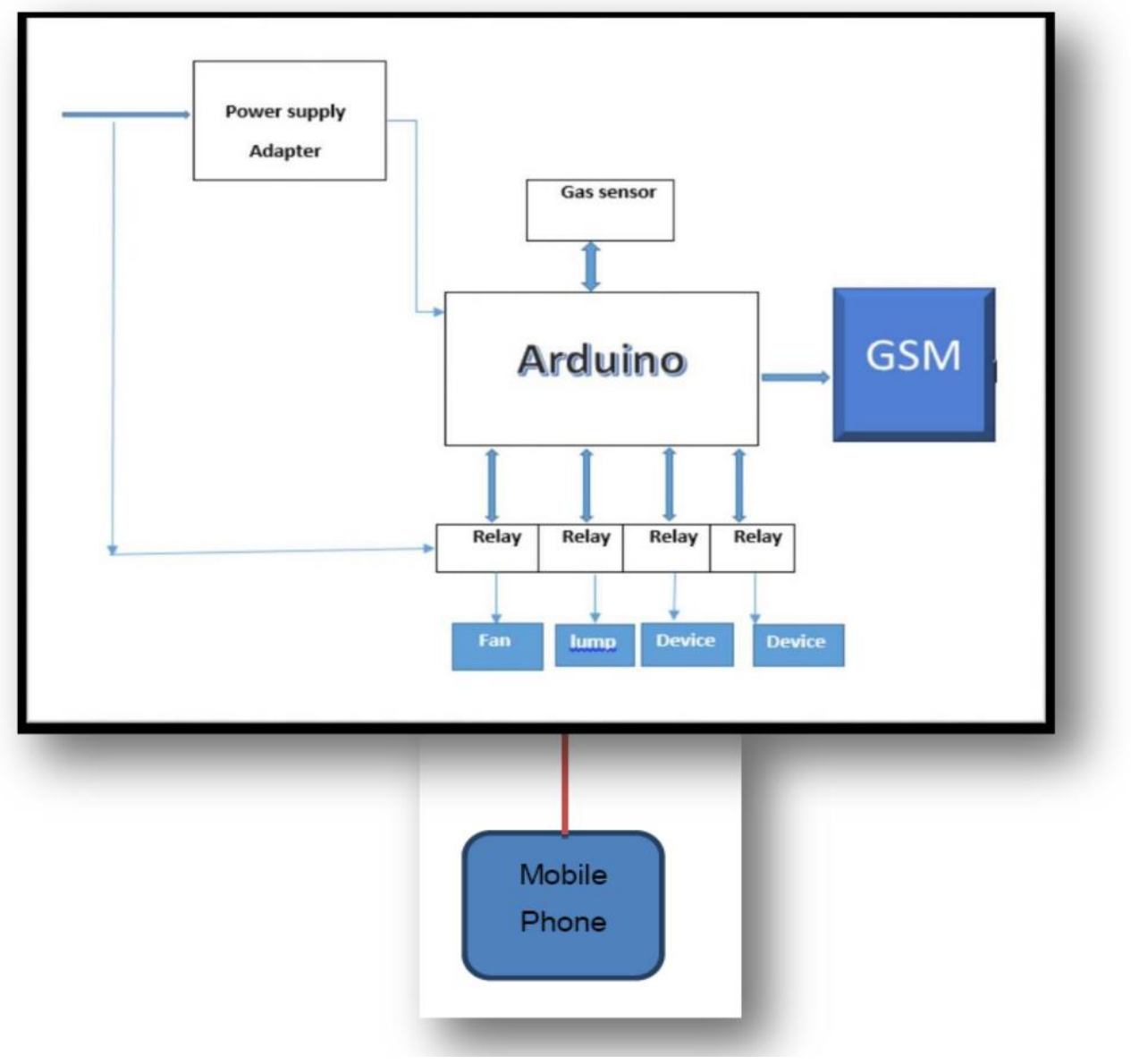

Fig.1. Block diagram of the system.

are continually processed by the microcontroller and an alert is sent to the mobile station when something happens. Besides getting the alert after reaching the limit, it is possible to know the temperature at home at any time by sending an instruction to the microcontroller. These units of the system are responsible for the security of a home. The other part of the microcontroller unit is the light control system which can be operated remotely using the mobile station. The user could get information about the state of light, whether it is ON or OFF, and control the state of light remotely from the mobile station.

The Sim900 GPRS/GSM module acts as the mediator between the microcontroller unit and the mobile station and is responsible for the communication between them. This unit is responsible for sending information from the microcontroller to the mobile station and for sending the instruction from the mobile station to the microcontroller. The instruction sent by the user from the mobile station is executed by the microcontroller. The second unit of the system is the mobile station which is just a mobile phone that does not require any special feature or any special application to be a part of the system. Any mobile phone supporting the messaging application is suitable. The instruction to the microcontroller is sent by using text messages and the alert from the microcontroller is received as a text as well. The system acts as a smart home system providing security to the home as well as providing a remote management system for the devices inside the home.

\section{A. Interfacing of Sim900 GPRS/GSM Module}

The Sim900 module is an important part of the system responsible for communication between the microcontroller and the mobile phone. ATtention (AT) commands are used to interface the module as well as to configure it. AT commands are inserted in C-language as a string of characters which are sent to the module using the terminal program.

\section{B. Interfacing and Implementation of Sensor}

There are different areas which have to be monitored frequently and devices which have to be checked. The doors and windows need to be monitored from burglars if they try to open them as well as the movement of strangers around the house premises should be monitored. Similarly, a member of the home might want to know the state of electronic devices after leaving home and might need to turn the devices OFF if they are left ON. In addition, the temperature of the home has to be monitored as well to trigger the alarm upon reaching the critical point. The 
monitoring of temperature, a stranger's movement, and the opening and closing of doors and windows are done by the designated sensors. The sensors could be implemented as different types of detectors according to the necessity of the application and the human desire. The operation of sensors is managed by software. Since there are different types of sensors, they are interfaced according to the output and properties of the sensor. The external circuit for interfacing the sensor to the application depends on its type.
The sensor and flame should keep a certain distance to avoid high temperature from damaging the sensor. The shortest test distance is $80 \mathrm{~cm}$, if the flame is bigger, test it with farther distance. The detection angle is 60 degrees so the flame spectrum is especially sensitive.

The microcontroller reads the output voltage of the sensor every second by using the function analogRead. Temperature is function of output voltage, and thus temperature can be calculated by using the mathematical formula. If it exceeds the limit defined in the software, it

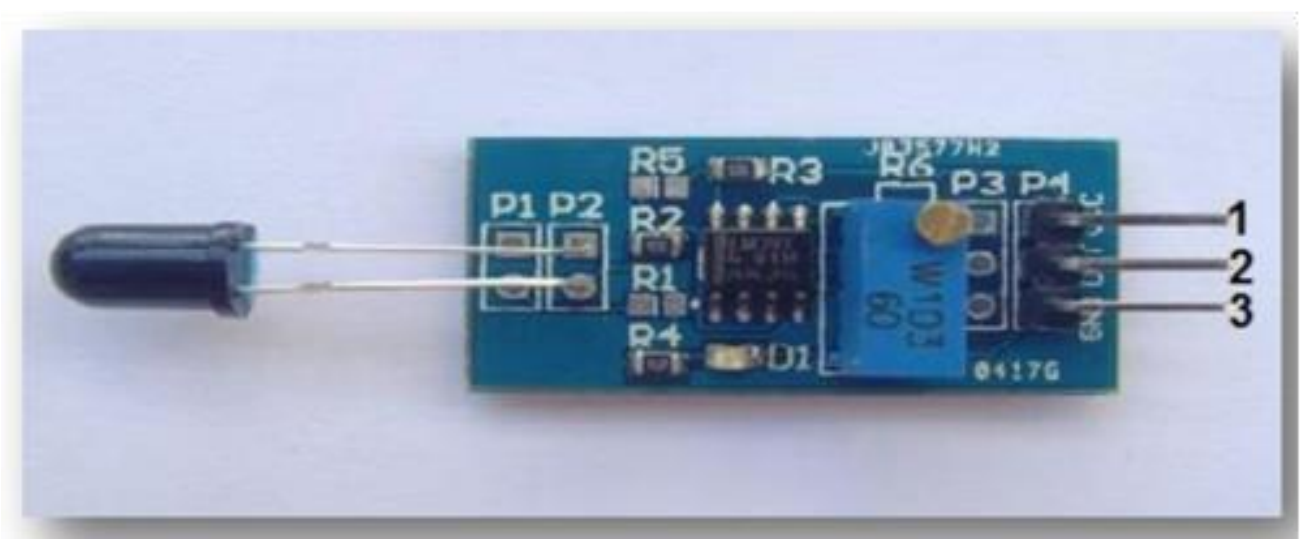

Fig.2. Heat sensor.

\section{Heat Sensor}

Heat (Flame) sensor shown in Fig. 2 is the most sensitive to ordinary light that is why its reaction is generally used as flame alarm purposes. This module can detect flame or wavelength in $760 \mathrm{~nm}$ to $1100 \mathrm{~nm}$ range of light source. Small plate output interface can and single-chip can be directly connected to the microcomputer IO port. will automatically send an SMS to the mobile phone specified in the software. The limit of the temperature to send the SMS can be changed depending on the environment of the place where the sensor is placed and on the application. In addition, it is possible to get the temperature of the place where the sensor is located by simply sending an SMS to the GPRS module... There are different ways to implement remotely managed electronic

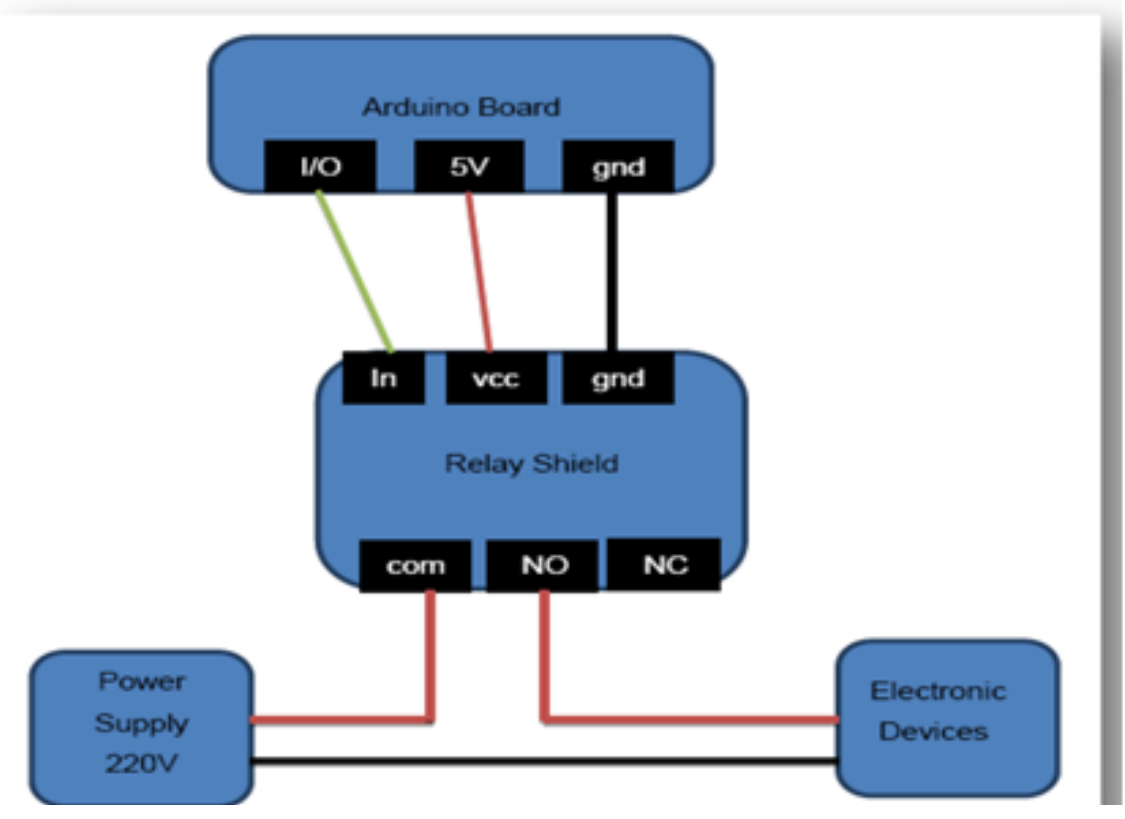

Fig. 3. Circuit diagram for the control of electronic devices. 
devices, for example management using the Ethernet, management using wireless devices or management using the GPRS/GSM module. The electronic device (the light control system) is managed remotely using the GPRS/GSM module (especially using the SMS) in this paper. Since the light uses a $220 \mathrm{~V} \mathrm{AC}$ current and the Arduino can provide only a $5 \mathrm{~V}$ DC current, a relay is used in between the Arduino and the light. The relay can be controlled by the I/O pins of the Arduino which finally controls the light. The circuit diagram to control the electronic devices using the $\mathrm{I} / \mathrm{O}$ pins of the Arduino Board via the relay shield is shown in Fig. 3.
The SH system triggers alarms to intrusion into the house through doors and windows as well as to the movement of a human being around the premises and around the restricted areas. The system also keeps surveillance over the temperature and triggered alarm upon reaching the critical point and above it. The whole system as the integration of the sensors and the light control system are implemented on Arduino platform using the Arduino Uno Board as shown in Fig. 4.

Fig. 4 presents the microcontroller unit with the Arduino board, GPRS shield, light and sensors. Power supply to the

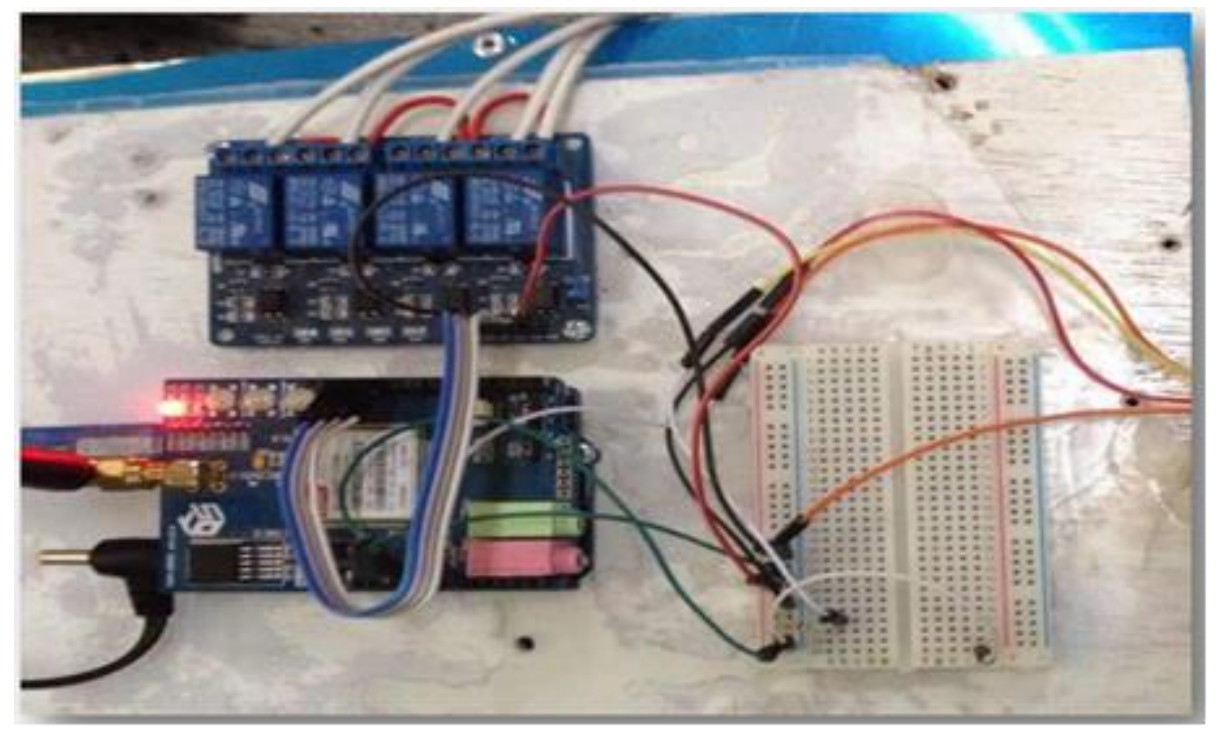

Fig. 4. Final system.

Basically, the electronic device can be managed remotely just by managing the $\mathrm{I} / \mathrm{O}$ pin of the Arduino board. To show the demo of the remotely managed light, a LED is used instead of the light bulb and while the light is controlled by the I/O pins of the Arduino via relays, the LED is controlled by the $\mathrm{I} / \mathrm{O}$ pins itself.

The code first checks the SMS messages and does the operation according to the content of the messages. The light will turn ON or OFF according to the instruction sent by the mobile phone. Upon the query of the status of light, an SMS is sent back to the mobile phone containing the status of light. In this way, the status of light can be known and the light can be turned ON or OFF remotely.

In this paper described $\mathrm{SH}$ system implementation is used one light control system along with three detectors: a temperature sensor as the heat detector, a passive infrared sensor as the motion detector, and a Hall Effect proximity sensor as the intrusion detector. The number of sensors used and the electronic devices managed remotely can be increased or decreased according to the necessity of the application. unit can be provided externally or from the USB port of the computer, and sensors and the GPRS shield drives the power from the unit. The whole system is implemented using the C-code language written on the Arduino platform. The software written on the platform can be uploaded to the microcontroller (i.e. the Arduino board) using Arduino IDE software.

The Arduino integrated development environment (IDE) is a cross-platform written in Java, whereas the programs are written in $\mathrm{C}$ or $\mathrm{C}++$. The platform comes with a software library along with the code editor and features such as syntax highlighting, brace matching, and automatic indentation. The whole program is written in the platform in the $\mathrm{C}$ language code which can be uploaded to the board by a simple upload button. Basically, the work is the integration of the software ( $\mathrm{C}$ language code) used to interface and implement the sensors, the GPRS module, and the remote management of the light.

\section{RESULTS}

The SH system has been implemented and the aim of the application to manage the electronic devices remotely has been achieved. 
TABLE 1 RESULTS OF THE INSTRUCTIONS AND THE RESPONSE TO AND FROM THE MOBILE STATION

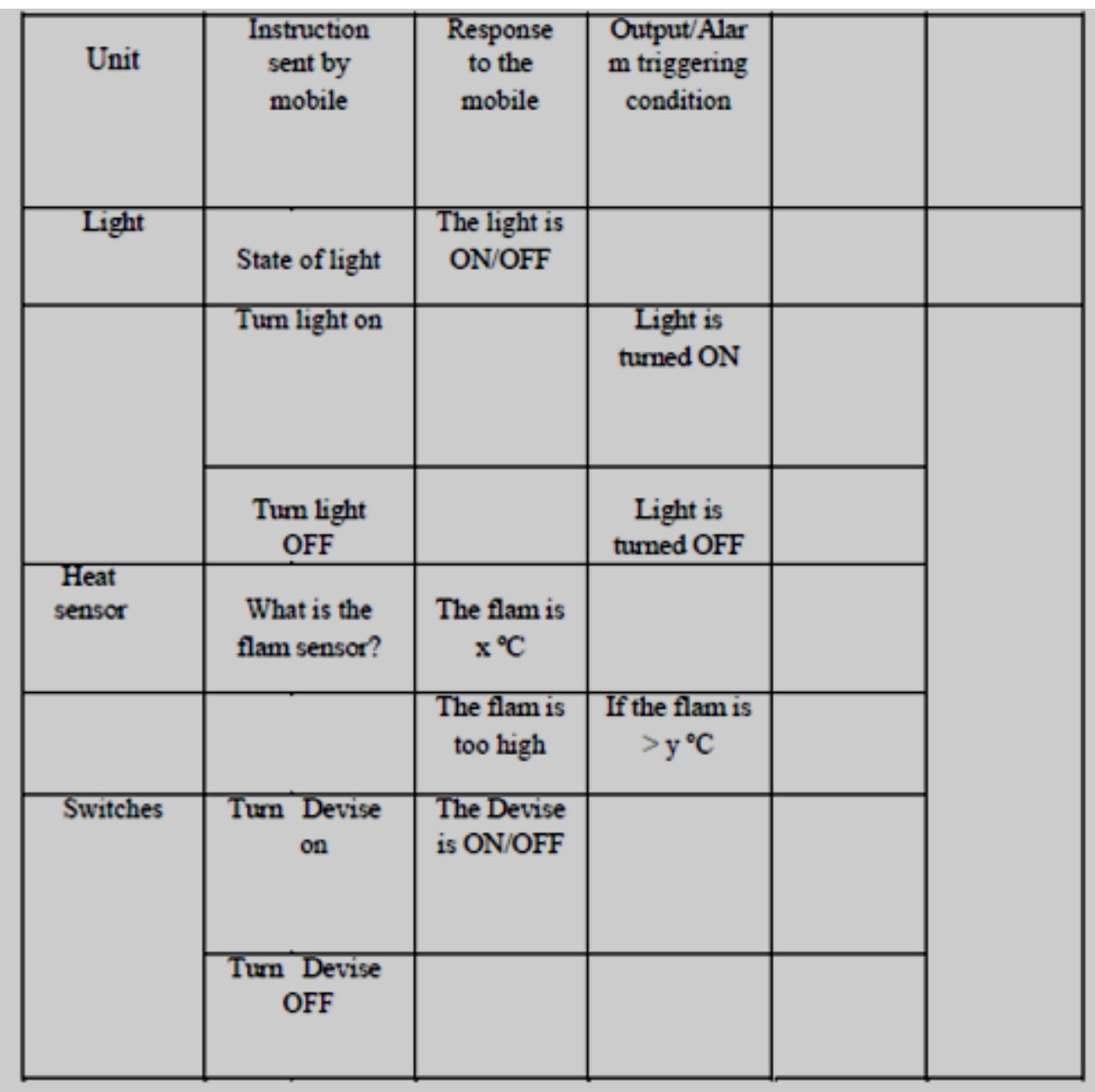

The microcontroller unit responds to the instructions sent by the mobile phone according to the necessity of the application as well as triggers the alarm upon a critical situation.

Table 1 shows the instructions sent to the microcontroller from the mobile station and output of the instructions as well as the response sent to the mobile station from the microcontroller respectively. As can be seen from Table 1, the instructions are sent only to the light system and the temperature sensor and they behave according to the instruction sent by the mobile phone.

Fig. 5 shows a screenshot of management of electronic devices (light control system) by the mobile station (the
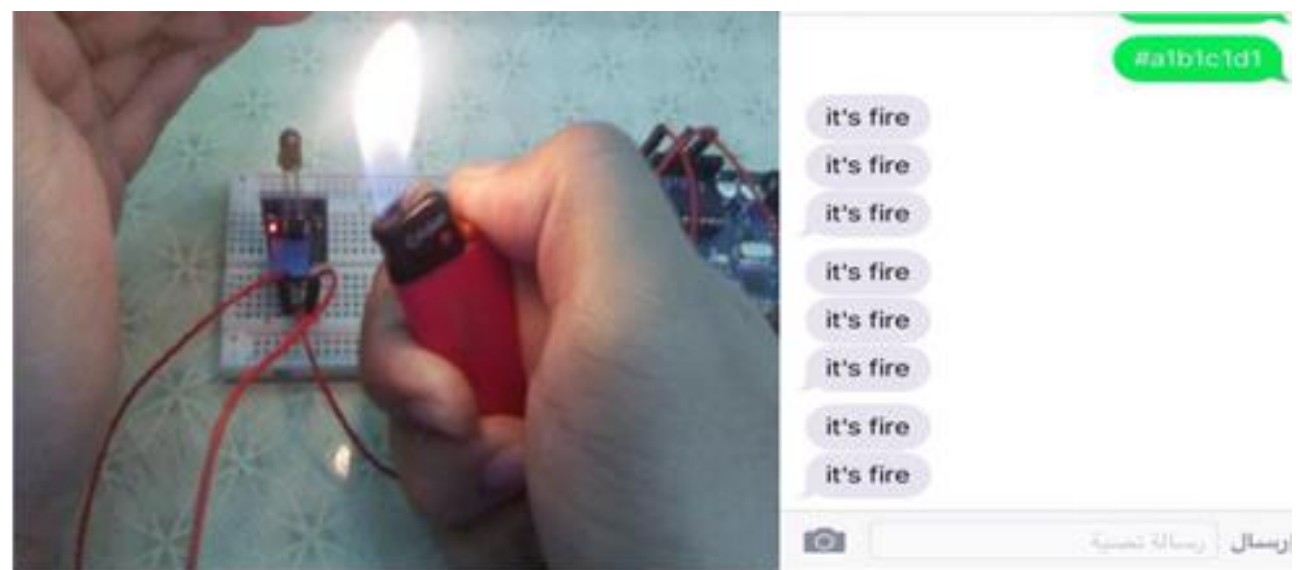

Fig. 5. Screenshot of the mobile station managing the light remotely. 
mobile phone). As can be seen the microcontroller sends a response to the mobile phone as an SMS mentioning whether the light is ON or OFF upon the instruction (state of light) sent by the mobile phone. The light can be turned $\mathrm{ON}$ and OFF by the SMS which can be visualized, and the response to the instruction is different when the light is ON or OFF...

\section{CONCLUSION}

The goal of the paper was to implement a smart home system by controlling the electronic devices at home remotely with a mobile phone and to receive alerts on intrusion and movement around restricted premises. The goal was achieved successfully. The devices were controlled by sending instructions as an SMS and the alerts were received as an SMS as well. A Hall Effect proximity sensor and a passive infrared sensor were used as detectors to detect the intrusion and movement around the restricted premises respectively. A temperature sensor was used as a heat detector and a LED light was used to show the demonstration of an electronic device management. Arduino Uno Board was used as a microcontroller while the SIM900 GPRS/GSM model was used for communication between the microcontroller unit and the mobile station. The mobile phone did not need to have any special application or hardware to be used as a mobile station. Any mobile phone supporting the SMS could be used as a mobile station.

\section{REFERENCES}

[1] K. Nisar, and A. Ibrahim, "A smart home model using android application," Proceedings of the 7th International Conference on Kansei Engineering and Emotion Research, Malaysia, 2018. https://doi.org/10.1007/978-981-10-8612-0 1

[2] M. Li, and W. Gu, "Smart home: architecture, technologies and systems," Procedia Computer Science, Vol.131, PP.393-400, 2018. https://doi.org/10.1016/j.procs.2018.04.219

[3] S. Darby, "Smart technology in the home: time for more clarity," Building Research and Information, Vol.46, Issue 1, PP.140-147, 2018. https://doi.org/10.1080/09613218.2017.1301707

[4] M. Al-Kuwari, and A. Ramadan, "Smart-home automation using IoT-based sensing and monitoring platform," 2018 IEEE 12th International Conference on Compatibility, Power Electronics and Power Engineering, Qatar, 2018.

https://doi.org/10.1109/CPE.2018.8372548 